\title{
No Visible Initiation of Pharyngeal Swallow at Any Location
}

National Cancer Institute

\section{Source}

National Cancer Institute. No Visible Initiation of Pharyngeal Swallow at Any Location. NCI

Thesaurus. Code C127224.

A finding of no visible initiation of pharyngeal swallow at any location. 